\title{
The Mediation Role of Self-Esteem for Self-Stigma on Quality of Life for People With Schizophrenia: A Retrospectively Longitudinal Study
}

\author{
Wen-Yi Huang, ${ }^{1}$ Shu-Ping Chen, ${ }^{2}$ Amir H. Pakpour, ${ }^{3,4}$ and Chung-Ying Lin ${ }^{5}$ \\ ${ }^{1}$ Department of Occupational Therapy, Jianan Psychiatric Center, Ministry of Health and Welfare, Tainan, Taiwan \\ 2 Department of Occupational Therapy, Faculty of Rehabilitation Medicine, University of Alberta, Edmonton, Canada \\ ${ }^{3}$ Social Determinants of Health Research Center, Qazvin University of Medical Sciences, Qazvin, Iran \\ ${ }^{4}$ Department of Nursing, School of Health and Welfare, Jönköping University, Jönköping, Sweden \\ ${ }^{5}$ Department of Rehabilitation Sciences, Faculty of Health and Social Sciences, The Hong Kong Polytechnic University, \\ Hung Hom, Hong Kong
}

\begin{abstract}
$B$ ackground: Among patients with schizophrenia, there is evidence of a negative association between self-stigma and subjective quality of life (SQoL), and self-esteem was an important mediator in the association. We attempted to use a longitudinal study to investigate the aforementioned mediation on a sample with schizophrenia. Methods: We used longitudinal data retrieved from medical records of a psychiatric centre between June 2014 and December 2015. In the data, we retrieved information of self-stigma using the Self-Stigma Scale - Short; SQoL, using the WHO questionnaire on the Quality of Life - Short Form; and self-esteem, using the Rosenberg Self-Esteem Scale. All the measures were evaluated five times. Linear mixed-effect models accompanied by Sobel tests were used to tackle the mediating effects. Results: Data from 74 patients (57 males) with schizophrenia were eligible for analysis; their mean (SD) age was 39.53 (10.67); mean age of onset was 22.95 (8.38). Self-esteem was a mediator for patients in physical $(p=.039)$, psychological $(p=.003)$, and social SQoL $(p=.004)$, but not in environment SQoL $(p=.051)$. Conclusion: Based on our findings, mental health professionals could tailor different programs to patients with schizophrenia, such as self-stigma reduction and self-esteem improvement programs. However, treatment as a whole should be sensitive to both self-stigma and selfesteem. Also, we should consider individuals' health and wellbeing from social perspectives of disability rather than the medical model of disability emphasising symptoms and medications.
\end{abstract}

Keywords: linear mixed-effect model, mediating effect, serious mental illness

People with schizophrenia usually suffer from their psychotic symptoms and impaired social functioning (American Psychiatric Association, 2013). Therefore, their health outcomes are generally lower than that of the general population (Hegarty, Baldessarini, Tohen, Waternaus, \& Oepen, 1994; Su, Ng, Yang, \& Lin, 2014). The subjective quality of life (SQoL) is one of the most important outcomes among the impaired health-related outcomes for people with schizophrenia, with growing interest for healthcare providers (Lin et al., 2012). Many studies (Carpenter \& Buchanan, 1994; Hegarty et al., 1994; Hooley, 2010; Yen et al., 2008) have shown that an individual's SQoL, including physical and psychological aspects, is impaired in people with a mental illness such as schizophrenia. Mental health professionals could improve the health and wellbeing of people with schizophrenia by finding ways to improve their SQoL.

An individual's SQoL is frequently appraised to assess his or her health and wellbeing (The WHOQOL Group, 1993). The World Health Organization (WHO) defines SQoL as a broad-ranging concept containing dimensions of physical health, psychological health, social relationships, and salient features in the individual's environment (The WHOQOL Group, 1993, 1998). Therefore, SQoL could be used as an indicator of health outcomes for people with mental illness. However, to address the SQoL of people with schizophrenia, mental health professionals need to know what factors determine the SQoL in such individuals. 
A person who belongs to a stigmatised group may suffer from self-stigma due to the awareness of the publicly held stigma regarding the group (Corrigan \& Watson, 2002). Self-stigma is one of the most important factors in an individual's SQoL (Lin, Chang, Wu, \& Wang, 2016). Although several theories have been proposed to conceptualise self-stigma (Chang, Lin, Gronholm, \& Wu, 2016; Corrigan, Watson, \& Barr, 2006; Livingston \& Boyd, 2010), there is a consensus of opinion that people with self-stigma have internalised a perceived stigma. In a patient with mental illness, self-stigma tends to reduce his/her motivation to accept treatment (Fung, Tsang, \& Corrigan, 2008; Tsang, Fung, \& Chung, 2010). Studies have demonstrated a causal relationship between an individual's self-stigma and SQoL (Chan \& Mak, 2014; Mashiach-Eizenberg, HassonOhayon, Yanos, Lysaker, \& Roe, 2013; Lin et al., 2016); others have shown that self-stigma negatively affects the self-esteem of a person with a mental illness (Corrigan \& Watson, 2002; Corrigan et al., 2006; Lysaker, Roe, Ringer, Gilmore, \& Yanos, 2012). As self-esteem has been found to mediate between self-stigma and many health-related outcomes such as depression and impaired social functioning (Yanos, Roe, \& Lysaker, 2010; Yanos, Roe, Markus, \& Lysaker, 2008), it is not surprising that researchers have proposed an evidence-based model of self-esteem as a mediating factor between self-stigma and an individual's SQoL (Mashiach-Eizenberg et al., 2013).

For several reasons we identified the need to reexamine the mediating role of self-esteem between the selfstigma in an individual and the individual's SQoL. First, to the best of our knowledge, the proposed evidence-based model has been tested only with a cross-sectional sample; that is, the casual relationships cannot be confirmed. Although we believe that the model can be supported using a longitudinal dataset, there is currently no such evidence. Second, most individuals with schizophrenia receive treatment, including medication, psychotherapy, and occupational therapy, and the treatments might have an impact on the proposed model. For example, one study found that relationships between self-stigma and SQoL were different between groups that engaged and groups that did not engage in community service programs (Huang \& Lin, 2015). However, we cannot tackle the treatment issue due to the nature of the cross-sectional design in the previous study (Mashiach-Eizenberg et al., 2013). Specifically, the treatment is dynamic, and its effects cannot be measured in a short period of time. Third, an individual's SQoL has physical, psychological, social, and environment domains (The WHOQOL Group, 1998), and the evidence-based model (Mashiach-Eizenberg et al., 2013) treats SQoL holistically rather than as an entity with different dimensions. The SQoL concept employed as a whole can provide mental health professionals with a full picture of an individual's health; however, a consideration of SQoL domains can help to illuminate specific health issues.

The purpose of this study was to examine the mediating role of self-esteem between self-stigma and SQoL using a cohort of individuals with schizophrenia. Instead of treating SQoL as a whole, we separately examined physical, psychological, social, and environment dimensions of SQoL.

\section{Methods \\ Subjects and Procedure}

A retrospective cohort study was conducted. Ethics approval was obtained from the Institutional Review Board of the Jianan Psychiatric Center (IRB \#15-011), and all subjects have provided oral consents in the study.

The inclusion criteria for eligible subjects included: (1) a diagnosis of schizophrenia based on medical records; (2) regular receipt of occupational therapy between March 2014 and December 2015 at the Jianan Psychiatric Center; (3) admission to the day-care centre of the Jianan Psychiatric Center, which indicates that the patient had no or little active psychotic symptoms; and (4) 20 to 75 years of age. Subjects were excluded if they had been admitted to any psychiatric hospital as an inpatient during March 2014 to December 2015. An occupational therapist at the Jianan Psychiatric Center first reviewed the medical records to identify eligible subjects; a research assistant then collected the data from the medical records of eligible subjects.

\section{Measures}

All measures were repeatedly evaluated every three to five months between June 2014 and December 2015. Selfstigma was measured at the following five time points: June, September and December 2014, and March and July 2015; self-esteem was measured five times: July and December 2014, and March, July and December 2015; SQoL was measured five times: September and December 2014, and March, July and December 2015. Since January 2014, the Department of Occupational Therapy, Jianan Psychiatric Center, routinely measured the self-stigma, selfesteem, and SQoL for day-care patients who participated in the program of occupational therapy. All the measures were self-reported with the assistance of an occupational therapist.

The Self-Stigma Scale - Short (SSS-S). The SSS-S, a selfrated questionnaire for measuring self-stigma of minority groups or people with mental illness, consists of nine items rated on a 4-point Likert scale, with a higher score indicating a higher level of self-stigma. The original SSSS (Mak \& Cheung, 2010) was developed in Hong Kong, with promising internal consistency according to Cronbach's alpha ( $\alpha=.81$ to .84$)$ and satisfactory construct validity (comparative fit index $[\mathrm{CFI}]=.97$ ). The SSS-S was adapted to a Taiwanese sample (Wu, Chang, Chen, Wang, \& Lin, 2015) with acceptable psychometric properties $(\alpha=.80$ to .91 ; CFI $=.99)$. The Cronbach's alpha of the SSS-S in the current study ranged from .878 to .952 in five repeated measures. 
The WHO Questionnaire on the Quality of Life - Short Form (WHOQOL-BREF). The original WHOQOL-BREF (The WHOQOL Group, 1998), a self-rated questionnaire for measuring SQoL across different populations (including healthy population and individuals with illness or disability), consists of 26 items with 2 general items and 24 items spread within four domains: physical, psychological, social, and environment. All items were rated on a 5-point Likert scale with a higher score indicating a higher level of SQoL. The WHOQOL-BREF was adapted to a Taiwanese population (Yao, Chung, Yu, \& Wang, 2002) with promising internal consistency ( $\alpha=.70$ to .91$)$ and testretest reliability ( $r=.76$ to .80 ). In addition, the Taiwan version of WHOQOL-BREF was found to have adequate internal consistency ( $\alpha=.68$ to .76$)$ and test-retest reliability (ICC $=.81$ to .86 ) in a Taiwanese sample with schizophrenia (Su et al., 2014). The Cronbach's alpha of the WHOQOL-BREF in the current study ranged from .934 to .962 in five repeated measures.

Rosenberg Self-Esteem Scale (RSES). The RSES, a selfrated questionnaire, consists of 10 items within one domain of self-esteem. All items are rated on a 4-point Likert scale. After scores of negatively worded items were reverse coded, a higher score suggested a higher level of self-esteem. The original RSES shows adequate internal consistency $(\alpha=.77)$ and test-retest reliability $(r=.63$ to .85; Byrne, 1983; Rosenberg, 1965; Silber \& Tippett, 1965), and the Taiwan version of the RSES was validated $(\mathrm{Wu}$, Lai, \& Shih, 2013). The Cronbach's alpha of the RSES in the current study ranged from .710 to .866 in five repeated measures.

\section{Data Analysis}

Several linear mixed-effect models adjusted for characteristics of subjects (age, sex, education, marital status, and receiving work training or not) were constructed to examine the direct effects of self-stigma and the mediating effects of self-esteem on the SQoL in individuals with schizophrenia receiving occupational therapy in a day-care centre. We constructed the linear mixed-effects model using all data with repeated measures. We applied a stationary model with the restricted maximum likelihood estimation in the linear mixed-effects model. In addition, the methods of $\mathrm{AR}(1)$ (i.e., autoregressive (1)) was used to tackle the variance-covariance matrix across the five repeated measures. We have ordered the five measures of each assessment in their temporal sequence. For example, self-stigma measured in June 2014 was assigned as the first measure, September 2014 as the second, December 2014 as the third, March 2015 as the fourth, and July 2015 as the fifth. The mixed-effects model informed us about the association between change in one variable and change in another variable. Therefore, using our longitudinal design, we could determine whether the change in self-stigma was associated with the change in self-esteem or the change in SQoL.

\begin{tabular}{lr}
\hline $\begin{array}{l}\text { Table } \mathbf{1} \\
\text { Participant Characteristics }\end{array}$ & \\
\hline Variable & $n(\%)$ \\
\hline${\text { Age }(\text { year })^{\dagger}}^{\dagger}$ Age of onset (year) ${ }^{\dagger}$ & $39.53 \pm 10.67$ \\
Body mass index $\left(\mathrm{kg} / \mathrm{m}^{2}\right)^{\dagger}$ & $22.95 \pm 8.38$ \\
Sex & $27.17 \pm 5.14$ \\
$\quad$ Male & \\
$\quad$ Female & $57(77.0)$ \\
Education & $17(23.0)$ \\
$\quad$ Elementary or below & \\
$\quad$ Junior high & $4(5.4)$ \\
$\quad$ Senior high & $16(21.6)$ \\
$\quad$ College or above & $39(52.7)$ \\
Marital status & $15(20.3)$ \\
$\quad$ Married & $15(20.3)$ \\
Single & $55(74.3)$ \\
Divorced & $4(5.4)$ \\
Work training & \\
$\quad$ Yes & $44(59.5)$ \\
$\quad$ No & $30(40.5)$ \\
\hline
\end{tabular}

Note: ${ }^{\dagger}$ presented as mean $\pm S D$.

The mediation models were examined using the following steps suggested by Baron and Kenny (1986) and Judd, Kenny, and McClelland (2001): (1) the effects of self-stigma on SQoL; (2) the effects of self-stigma on selfesteem; (3) the effects of self-esteem on SQoL. If steps 2 and 3 showed that self-stigma significantly affected selfesteem and self-esteem significantly affected SQoL, Sobel tests (Sobel, 1982) were used to further test the statistical significance of the mediation. In addition, we controlled for age, sex, education, marital status, and work training in the linear mixed-effects models.

All the statistical analyses were performed using SPSS 23.0 (IBM Corp., Armonk, NY).

\section{Results}

After identifying eligible subjects, the data from $74 \mathrm{pa}-$ tients with schizophrenia and a median age of 39.53 years $(S D=10.67)$ were used for analyses. The mean age of onset was $22.95(S D=8.38)$. Seventy-seven percent of the participants were male $(n=57) ; 73 \%$ had graduated from senior high or above $(n=54) ; 74 \%$ were single $(n=55)$. In addition to receiving occupational therapy services, $59.5 \%$ of the participants $(n=44)$ were enrolled in a sheltered employment program (Table 1). Moreover, the internal consistency of each instrument used in the study across five assessment points is reported in Table 2.

The impacts of self-stigma on subjects' SQoL were negative and significant throughout the four domains: physical $(B=-0.79, S E=0.31$; standardised $B=-0.22$; $p=.013)$, psychological $(B=1.15, S E=0.43$; standardised $B=-0.28 ; p=.009)$, social $(B=-1.28$, $S E=0.54 ;$ standardised $B=-0.28 ; p=.020)$, and environment $(B=-0.99, S E=0.46$; standardised $B=-0.22 ; p=.034)$. Also, self-stigma had a negative and significant effect on self-esteem $(B=-1.17$, $S E=0.53 ;$ standardised $B=-0.17 ; p=.029)$. The 


\begin{tabular}{llllll}
\hline $\begin{array}{l}\text { Table } \mathbf{2} \\
\text { Internal Consistency (Crobach's } \alpha \text { ) of the Three Instruments in this Study }\end{array}$ & & \\
\hline & Time 1 & Time 2 & Time 3 & Time 4 & Time 5 \\
\hline SSS-S & 0.884 & 0.879 & 0.952 & 0.878 & 0.913 \\
RSEM & 0.795 & 0.810 & 0.866 & 0.821 & 0.710 \\
WHOQOL-BREF & 0.949 & 0.959 & 0.962 & 0.949 & 0.934 \\
Physical & 0.676 & 0.800 & 0.743 & 0.800 & 0.700 \\
Psychological & 0.765 & 0.807 & 0.848 & 0.809 & 0.814 \\
Social & 0.789 & 0.903 & 0.876 & 0.798 & 0.657 \\
Environment & 0.911 & 0.923 & 0.939 & 0.890 & 0.860
\end{tabular}

Note: SSS-S = Self-Stigma Scale — Short; RSES = Rosenberg Self-Esteem Scale; WHOQOL-BREF = WHO Questionnaire on the Quality of Life - Short Form.

\section{Table 3}

Linear Mixed-Effects Models in Which Self-Stigma and Self-Esteem Were Constructed to Explain Subjective Quality of Life (SQLL)

\begin{tabular}{|c|c|c|c|c|c|c|}
\hline \multirow[b]{2}{*}{ Dependent variable } & \multicolumn{2}{|c|}{ Self-stigma $^{\dagger}$} & \multicolumn{2}{|c|}{ Self-esteem ${ }^{\dagger}$} & \multicolumn{2}{|c|}{ Sobel test } \\
\hline & $B(S E)$ & $p$ & $B(S E)$ & $p$ & $z$ & $p$ \\
\hline Self-esteem & $-1.17(0.53)$ & .029 & - & - & - & - \\
\hline Physical SQoL & $-0.79(0.31)$ & .013 & $0.23(0.04)$ & $<.001$ & 2.06 & .039 \\
\hline Psychological SQoL & $-1.15(0.43)$ & .009 & $0.36(0.04)$ & $<.001$ & 2.14 & .003 \\
\hline Social SQoL & $-1.28(0.54)$ & .020 & $0.29(0.06)$ & $<.001$ & 2.01 & .004 \\
\hline Environment SQoL & $-0.99(0.46)$ & .034 & $0.25(0.06)$ & $<.001$ & 1.95 & .051 \\
\hline
\end{tabular}

Note: $B=$ coefficient; $S E$ = standard error.

${ }^{\dagger}$ Adjusted for age, sex, education, marital status, and work training.

impacts of self-esteem on subjects' SQoL were positive and significant throughout the four domains: physical $(B=0.23, S E=0.04$; standardised $B=0.43 ; p<.001)$, psychological $(B=0.36, S E=0.04$; standardised $B=0.60$; $p<.001)$, social $(B=0.29, S E=0.06$; standardised $B=0.43$; $p<.001)$, and environment $(B=0.25, S E=0.06$; standardised $B=0.38 ; p<.001)$. The mediated effects of self-esteem were shown in the relationships between self-stigma and all but the environment domain of SQoL. However, the mediated effect of self-esteem in the relationship between self-stigma and environment domains of SQoL was very close to significant $(p=.051)$. This mediated effect was supported by the Baron and Kenny's (1986) methods: effect of self-stigma on self-esteem $(p=.029)$ and that of self-esteem on environment SQoL $(p<.001)$ were both supported (Table 3).

\section{Discussion}

Using a retrospective longitudinal design, our results support the mediation model in which the SQoL is impacted by self-stigma through self-esteem for individuals with schizophrenia who regularly receive occupational therapy; specifically, self-esteem mediated SQoL in all domains, except for environment domain.

Our results agree with previous findings that selfstigma reduces the self-esteem of an individual with mental illness (Corrigan \& Watson, 2002; Corrigan et al., 2006; Lysaker et al., 2012). Also, our findings were aligned with studies that found negative impacts of self-stigma on an individual's SQoL (Chan \& Mak, 2014; Mashiach-Eizenberg et al., 2013; Lin et al., 2016). In addition, our results agree with the mediation model proposed by MashiachEizenberg et al. (2013) that self-esteem is a mediating factor between an individual's self-stigma and his or her SQoL. However, the mediated effect of self-esteem in the relationship between self-stigma and environment SQoL was not fully supported by the Sobel test. A possible explanation is the role of self-esteem under different cultures (Brown, Cai, Oakes, \& Deng, 2009; Heine, Lehman, Markus, \& Kitayama, 1999). A controversy is that different cultures may not value self-esteem similarly. It seems that East Asians had lower self-esteem as compared with North Americans (Heine et al., 1999). However, Brown et al. (2009) suggested that self-esteem is universally important across the two countries of America and Taiwan. Based on our findings, we agree with Brown et al. (2009) that self-esteem does play an important role in psychological outcomes; however, the effect of self-esteem varies in different psychological outcomes. Specifically, the environment SQoL, which assesses the primary living conditions of an individual, may have diminished association with self-esteem when there is another stronger predictor (i.e., self-stigma). That is, self-stigma may contribute more than self-esteem does to the environment SQoL due to unfriendly treatment perceived in the environment.

Our results indicated two issues in the mediation models that have never been elaborated. In our study, mediation models were supported for the physical, psychological, and social domains but not for the environment domain of an individual's SQoL. Some studies (Chan \& Mak, 2014; Mashiach-Eizenberg, 2013) treated the SQoL as a unidimensional concept, which is beneficial when mental health professionals want to evaluate an 
individual's overall health and wellbeing. However, we suggest that using different dimensions of SQoL can help mental health professionals design interventions to address specific needs of people with schizophrenia. Using our results as examples, a healthcare provider might provide a self-stigma reduction program that would work more effectively than a self-esteem improvement program to improve the environment domain of an individual's SQoL, because we found that self-esteem had less impact on the environment SQoL for individuals with schizophrenia.

Our findings have two major implications. First, self-stigma reduction programs, such as Narrative Enhancement and Cognitive Therapy (Yanos, Roe, \& Lysaker, 2011; Yanos et al., 2012), could be beneficial for people with schizophrenia. A self-stigma reduction program may prevent them from the worst health outcomes. Second, self-esteem improvement programs empowering people with schizophrenia (Lecomte et al., 1999) may work together with the self-stigma reduction program to maximise the improvement of health-related outcomes. Moreover, metacognitive oriented treatments may be a promising intervention to tackle self-esteem and self-stigma because such treatments could assist meaning making and guide alternative ways of thinking about the self and life challenges. However, it should be appreciated that either interventions on self-stigma or self-esteem should be viewed as a whole. Interventions must consider individuals' health and wellbeing from social perspectives of disability rather than the medical model of disability emphasising symptoms and medications.

There were some limitations in our study. First, in addition to occupational therapy, patients received other interventions and therapies at the day-care centre. We were unable to control for such diverse interventions in the study due to the relatively small sample size. To corroborate our study findings, future studies could use a larger sample size to control therapeutic interventions. Second, not all the subjects in our study were inpatients and the generalisability of our results may thus be limited. Specifically, outpatients have more contact with the communities more do inpatients, and the SQoL and stigma may differ between inpatients and outpatients. Third, as we only recruited participants who were not in an early phase of mental illness in Taiwan, the generalisability of our study results might be restricted. Specifically, we cannot ensure that similar findings will be found in other cultures, especially the Western culture with its focus on individualism and strong selfesteem (Ali, Lee, Hsieh, \& Krishnan, 2005; Heine et al., 1999), or in other types of mental illness. Future studies are thus warranted to corroborate our findings. Fourth, although we have ordered our assessment time points to align with hypothesised temporal associations (i.e., the assessment on self-stigma was first, followed by self-esteem and SQoL), self-esteem and SQoL were assessed at the same time for the last four assessments. Therefore, the temporal associations between self-stigma and the other two measures (self-esteem and SQoL) were strong, and the temporal association between self-esteem and SQoL was weak. Lastly, and importantly, the measurement invariance across time (i.e., the five assessment time points) was not examined in our study because of the small sample size. As an important assumption in the linear mixedeffects model is time invariance for all the measures (i.e., we used the stationary-based model), our findings might be somewhat biased if such an assumption is not fulfilled.

In conclusion, self-esteem mediated the causal relationship between self-stigma and the physical and psychological domains of the SQoL in people with schizophrenia. Mental health professionals could tailor programs to patients with schizophrenia by providing self-stigma reduction and self-esteem improvement programs.

\section{Conflict of Interest}

None.

\section{References}

Ali, A.J., Lee, M., Hsieh, Y.C., \& Krishnan, K. (2005). Individualism and collectivism in Taiwan. Cross Culture Management: An International Journal, 12, 3-16. https://doi.org/10.1108/13527600510798105

American Psychiatric Association. (2013). Diagnostic and statistical manual of mental disorders (5th ed.). Washington, DC: Author.

Baron, R.M., \& Kenny, D.A. (1986). The moderator-mediator variable distinction in social psychological research: Conceptual, strategic and statistical considerations. Journal of Personality and Social Psychology, 51, 1173-1182. doi:10.1037/0022-3514.51.6.1173

Brown, J.D., Cai, H., Oakes, M.A., \& Deng, C. (2009), Cultural similarities in self-esteem functioning: East is East and West is West, but sometimes the Taiwan do met. Journal of Cross-Cultural Psychology, 40, 140-157. https://doi.org/10.1177/0022022108326280

Byrne, B.M. (1983). Investigating measures of self-concept. Measurement and Evaluation in Guidance, 16, 115-126.

Carpenter, W.T., \& Buchanan, R.W. (1994). Schizophrenia. The New England Journal of Medicine, 330, 681-690. doi:10.1056/NEJM199403103301006

Chan, K.K.S., \& Mak, W.W.S. (2014). The mediating role of self-stigma and unmet needs on the recovery of people with schizophrenia living in the community. Quality of Life Research, 23, 2559-2568. doi:10.1007/s11136-014-0695-7

Chang, C.-C., Lin, C.-Y., Gronholm, P.C., \& Wu, T.-H. (2016). Cross-validation of two commonly used self-stigma measures, Taiwan versions of the Internalized Stigma Mental Illness scale and Self-Stigma Scale-Short, for people with mental illness. Assessment. Advance online publication. doi:10.1177/1073191116658547

Corrigan, P.W., \& Watson, A.C. (2002). The paradox of selfstigma and mental illness. Clinical Psychology: Science and Practice, 9, 35-53. doi:10.1093/clipsy.9.1.35

Corrigan, P.W., Watson, A.C., \& Barr, L. (2006). The selfstigma of mental illness: Implications for self-esteem and 
self-efficacy. Journal of Social and Clinical Psychology, 25, 875-884. doi:10.1521/jscp.2006.25.8.875

Fung, K.M.T., Tsang, H.W.H., \& Corrigan, P.W. (2008). Selfstigma of people with schizophrenia as predictor of their adherence to psychosocial treatment. Psychiatric Rehabilitation Journal, 32, 95-104. doi:10.2975/32.2.2008.95.104

Hegarty, J.D., Baldessarini, R.J., Tohen, M., Waternaus, C., \& Oepen, G. (1994). One hundred years of schizophrenia: A meta-analysis of the outcomes literature. The American Journal of Psychiatry, 151, 1409-1416. doi:10.1176/ajp.151.10.1409

Heine, S.J., Lehman, D.R., Markus, H.R., \& Kitayama, S. (1999). Is there a universal need for positive self-regard? Psychological Review, 106, 766-794. https://doi.org/10.1037/0033-295X.106.4.766

Hooley, J.M. (2010). Social factors in schizophrenia. Current Directions in Psychological Science, 19, 238-242. doi:10.1177/0963721410377597

Huang, W.-Y., \& Lin, C.-Y. (2015). The relationship between self-stigma and quality of life among people with mental illness who participated in a community program. Journal of Nature and Science, 1, e135.

Judd, C.M., Kenny, D.A., \& McClelland, G.H. (2001). Estimating and testing mediation and moderation in within-subject designs. Psychological Methods, 6, 115-134. https://doi.org/10.1037/1082-989X.6.2.115

Lecomte, T., Cyr, M., Lesage, A.D., Wilde, J., Leclerc, C., \& Ricard, N. (1999). Efficacy of a self-esteem module in the empowerment of individuals with schizophrenia. The Journal of Nervous \& Mental Disease, 187, 406-413. doi:10.1097/00005053-199907000-00003

Lin, C.-Y., Chang, C.-C., Wu, T.-H., \& Wang, J.-D. (2016). Dynamic changes of self-stigma, quality of life, somatic complaints, and depression among people with schizophrenia: A pilot study applying kernel smoothers. Stigma and Health, 1, 29-43. doi:10.1037/sah0000014

Lin, C.-Y., Luh, W.-M., Yang, A.-L., Su, C.-T., Wang, J.-D., \& Ma, H.-I. (2012). Psychometric properties and gender invariance of the Chinese version of the self-report pediatric quality of life inventory version 4.0: short form is acceptable. Quality of Life Research, 21, 177-182. doi:10.1007/s11136-011-9928-1

Livingston, J.D., \& Boyd, J.E. (2010). Correlates and consequences of internalized stigma for people living with mental illness: A systematic review and metaanalysis. Social Science \& Medicine, 71, 2150-2161. doi:10.1016/j.socscimed.2010.09.030

Lysaker, P.H., Roe, D., Ringer, J., Gilmore, E.M., \& Yanos, P.T. (2012). Change in self-stigma among persons with schizophrenia enrolled in rehabilitation: Associations with self-esteem and positive and emotional discomfort symptom. Psychological Services, 9, 240-247. doi:10.1037/a0027740

Mak, W.W.S., \& Cheung, R.Y.M. (2010). Self-stigma among concealable minorities in Hong Kong: Conceptualization and unified measurement. American Journal of Orthopsychiatry, 80, 267-281. doi:10.1111/j.1939-0025.2010.01030.x

Mashiach-Eizenberg, M., Hasson-Ohayon, I., Yanos, P.T., Lysaker, P.H., \& Roe, D. (2013). Internalized stigma and quality of life among persons with severe mental illness: The mediating roles of self-esteem and hope. Psychiatry Research, 208(1), 15-20. doi:10.1016/j.psychres.2013.03.013

Rosenberg, M. (1965). Society and the adolescent self-image. Princeton, NJ: Princeton University Press.

Silber, E., \& Tippett, J.S. (1965). Self-esteem: Clinical assessment and measurement validation. Psychological Report, 16, 10171071. doi:10.2466/pr0.1965.16.3c.1017

Sobel, M.E. (1982). Asymptotic confidence intervals for indirect effects in structural equation models. In S. Leinhardt (Ed.), Sociological methodology (pp. 290-312). Washington, DC: American Sociological Association.

Su, C.-T., Ng, H.-S., Yang, A.-L., \& Lin, C.-Y. (2014). Psychometric evaluation of the Short Form 36 Health Survey (SF-36) and the World Health Organization Quality of Life Scale Brief Version (WHOQOL-BREF) for patients with schizophrenia. Psychological Assessment, 26, 980-989. doi:10.1037/a0036764

The WHOQOL Group. (1993). Study protocol for the World Health Organization project to develop a Quality of Life assessment instrument (WHOQOL). Quality of Life Research, 2, 153-159. doi:10.1007/BF00435734

The WHOQOL Group. (1998). Development of the World Health Organization WHOQOL-BREF quality of life assessment. Psychological Medicine, 28, 551-558. doi:10.1017/S0033291798006667

Tsang, H.W.H., Fung, K.M.T., \& Chung, R.C.K. (2010). Selfstigma and stages of change as predictors of treatment adherence of individuals with schizophrenia. Psychiatry Research, 180, 10-15. doi:10.1016/j.psychres.2009.09.001

Wu, P.-H., Lai, Y.-C., \& Shih, C.-L. (2013). Evaluation of the Rosenberg Self-esteem Scale using the rating scale model [in Chinese]. Psychological Testing, 60, 263-289.

Wu, T.-H., Chang, C.-C., Chen, C.-Y., Wang, J.-D., \& Lin, C.-Y. (2015). Further psychometric evaluation of the Self-Stigma Scale-Short: Measurement invariance across mental illness and gender. PLoS One, 10, e0117592. doi:10.1371/journal.pone. 0117592

Yao, G., Chung, C.-W., Yu, C.-F., \& Wang, J.-D. (2002). Development and verification of validity and reliability of the WHOQOL-BREF Taiwan version. Journal of the Formosan Medical Association, 101, 342-351.

Yanos, P.T., Roe, D., Markus, K., \& Lysaker, P.H. (2008). Pathways between internalized stigma and outcomes related to recovery in schizophrenia-spectrum disorders. Psychiatric Services, 59, 1437-1442. doi:10.1176/ps.2008.59.12.1437

Yanos, P.T., Roe, D., \& Lysaker, P.H. (2010). The impact of illness identity on recovery from severe mental illness. American Journal of Psychiatric Rehabilitation, 13, 73-93. doi:10.1080/15487761003756860

Yanos, P.T., Roe, D., \& Lysaker, P.H. (2011). Narrative enhancement and cognitive therapy: A new group-based treatment for internalized stigma among persons with severe mental illness. International Journal of Group Psychotherapy, 61, 577-595. doi:10.1521/ijgp.2011.61.4.576

Yanos, P.T., Roe, D., Lysaker, P.H., West, M.L., \& Smith, S.M. (2012). Group-based treatment for internalized stigma among persons with severe mental illness: Findings from a 
randomized controlled trial. Psychological Services, 9, 248258. doi:10.1037/a0028048

Yen, C.-F., Cheng, C.-P., Huang, C.-F., Yen, J.-Y., Ko, C.-H., \& Chen, C.-S. (2008). Quality of life and its association with insight, adverse effects of medication and use of atypical antipsychotics in patients with bipolar disorder and schizophrenia in remission. Bipolar Disorders, 10, 617-624. doi:10.1111/j.1399-5618.2007.00577.x 